\title{
Debye-Waller Factor in an Isotopically Disordered Semiconductor Crystal
}

\author{
S.C. Gairola* \\ Department of Physics (School of Sciences), H.N.B. Garhwal University (A Central University) Campus Pauri, \\ Pauri-246001, Uttarakhand, India
}

(Received March 16, 2015; revised version April 27, 2016; in final form May 24, 2016)

The diagonal and non-diagonal parts for the Debye-Waller factor have been established using equation of motion technique of quantum dynamics and the Dyson equation approach. The double time temperature dependent phonon Green function has been taken to find the phonon linewidth and phonon shift. Renormalized mode frequency has been investigated in terms of electron-phonon coupling constant and temperature. The effect of electron-phonon interaction on the Debye-Waller factor has been studied in low temperature limit in low impurity concentration in semiconductor crystals.

DOI: $10.12693 /$ APhysPolA.129.1141

PACS/topics: 63.70.+h, 87.64.Bx, 63.20.-e, 63.20.Pw, 64.70.kg

\section{Introduction}

The widespread applications of semiconductor technology have become a renaissance in 21st century to develop new materials. The work on bulk semiconductor crystals has been carried out recently [1]. The large amount of work is being done on different semiconductor materials according to their utility towards society [2-11]. It is known that like X-rays, thermal neutrons have a de Broglie wavelength of the order of the interatomic spacings in crystals so that they can be used in diffraction studies [12]. The Debye-Waller factor (DWF) is used to find the differential scattering cross-section through dynamical structure factor in neutron scattering in impure anharmonic solids [13]. The Debye-Waller factor is one of the dynamical properties of the crystals, and its pressure dependence is found in silicon and germanium by using statistical moment method [14].

The quantity $[2 \mathrm{~W}]$ is called the Debye-Waller factor which governs the temperature dependence of the scattering cross-section [15]. It is determined in compound semiconductors by utilizing a recently proposed eight-parameter bond-bending force model [16] and in ternary chalcopyrite semiconductors from the available bulk modulus, and interionic separation distances [17]. The progress of research in semiconductor physics is achieved due to its (DWF) use to find the magnetic form factor of $\mathrm{Eu}^{2+}$ in $\mathrm{EuS}$ [18], local structural properties in the terahertz semiconductor $\mathrm{Zn}_{1-x} \mathrm{Cd}_{x} \mathrm{Te}$ [19], to describe observed band gap dependence [20], thermodynamic properties of semiconductor compounds [21], and vibrational dynamics and band structure of methylterminated Ge(111) [22]. The temperature dependence of DWF is governed by the molecular dynamics study

*e-mail: dgsureshc@yahoo.co.in; scgairola@rediffmail.com in InSb [23], calculated from ab initio force constants in sphalerite III-V semiconductors [24], and also obtained from statistical moment method in compound semiconductors [25]. In addition to these works, effect of anharmonicity has been applied to calculate different cumulants. The second cumulant gives DWF which is dependent on temperature [26-30].

It is established fact that theory based upon harmonic approximation fails to explain the experimental observed crystal properties due to zero point vibration even at zero temperature. This can be understood by taking impurity into consideration. This changes the frequency spectrum of host crystal. This creates gap, localized and resonance modes [31-34]. The presence of electron in crystal tries to distort the lattice causes to increase its effective mass. This distortion of the lattice is limited to the immediate vicinity of the electron (small polaron) or extends over several lattice constants (large polaron) [35]. Polaron state occurs up to second order in electronic heat capacity in low temperature limit [36]. An electron interacts with harmonic mode and localized mode which is responsible for the formation of many body system in semiconductor continuum.

According to Hall et al. electron can absorb a photon by indirect transition to conserve the energy and momentum laws at the same time [37]. A double time temperature dependent phonon Green function is taken to solve the present problem with the help of Hamiltonian contributed by harmonic part, electron part, electronphonon interaction part, and defect part. The work has been done on neutron scattering in impure anharmonic solids [13], but in present case electron-phonon interaction is taken in semiconductor crystals with isotopic impurity in low concentration in low temperature limit to develop the theory of DWF. The present work differs from previous work that they have not taken the electron-phonon interaction using equation of motion technique of quantum dynamics, and the Dyson equation 
approach in their studies. The effect of electron-phonon coupling constant, and temperature have been investigated in detail. The DWF has been separated into diagonal and non-diagonal parts in semiconductor crystals.

This paper is divided into six sections. These are introduction, formulation of the problem, evaluation of the Fourier transformed phonon Green function, phonon linewidth, and phonon shift, evaluation of renormalized mode frequency and Debye-Waller factor, results and discussions, and conclusion of the theory at the end.

\section{Formulation of the problem}

The theory of DWF $2 \mathrm{~W}$ can be achieved for semiconductor crystals by taking the following expression as $[12,13,38,39]$ :

$$
\begin{aligned}
& 2 W=-(M N)^{-1} \sum_{k k^{\prime}}[\boldsymbol{K} \cdot \boldsymbol{e}(k)]\left[\boldsymbol{K} \cdot \boldsymbol{e}\left(k^{\prime}\right)\right] \\
& \times\left(\varepsilon_{k} \varepsilon_{k^{\prime}}\right)^{-1 / 2} \exp \left(2 \pi \mathrm{i}\left(\boldsymbol{k}+\boldsymbol{k}^{\prime}\right) \cdot \boldsymbol{x}(l)\right) \\
& \quad \times \lim _{\omega \rightarrow 0} \int_{-\infty}^{\infty} n(\varepsilon) \mathrm{d} \varepsilon \operatorname{Im} G_{k k^{\prime}}(\varepsilon+\mathrm{i} \omega) .
\end{aligned}
$$

In above Eq. (1), $n(\varepsilon), G_{k k^{\prime}}(\varepsilon)$ represent the Planck distribution function and the Fourier transformed double time temperature dependent phonon Green function, respectively $[12,13,38,39]$. Thus, it is clear that the evaluation of imaginary part of the phonon Green function is the main focus to achieve our goal.

\section{Evaluation of the Fourier transformed phonon Green function, phonon linewidth, and phonon shift}

The present theory is developed by taking Hamiltonian as [40-49]:

$$
H=H_{\mathrm{op}}+H_{\mathrm{oe}}+H_{\mathrm{D}}+H_{\mathrm{ep}} .
$$

In above Eq. (2), $H_{\mathrm{op}}, H_{\mathrm{oe}}, H_{\mathrm{D}}, H_{\mathrm{ep}}$ represent harmonic part, electron part, defect part, and electron-phonon interaction part of Hamiltonian, respectively. They are expressed as

$$
\begin{aligned}
& H_{\mathrm{op}}=(\hbar / 4) \sum_{k} \varepsilon_{k}\left(A_{k}^{*} A_{k}+B_{k}^{*} B_{k}\right), \\
& H_{\mathrm{oe}}=\hbar \sum_{q} \varepsilon_{q} b_{q}^{*} b_{q} \\
& H_{\mathrm{D}}=-\hbar \sum_{k_{1}, k_{2}}\left[C\left(\boldsymbol{k}_{1}, \boldsymbol{k}_{2}\right) B_{k_{1}} B_{k_{2}}\right. \\
& \left.\quad-D\left(\boldsymbol{k}_{1}, \boldsymbol{k}_{2}\right) A_{k_{1}} A_{k_{2}}\right] \\
& H_{\mathrm{ep}}=g \hbar \sum_{k, q} b_{Q}^{*} b_{q} B_{k}
\end{aligned}
$$

where

$$
\begin{aligned}
& A_{k}=a_{k}+a_{-k}^{*}=A_{k}^{*}, \quad B_{k}=a_{k}-a_{-k}^{*}=-B_{-k}^{*}, \\
& \boldsymbol{Q}=\boldsymbol{k}+\boldsymbol{q} .
\end{aligned}
$$

In above $b_{q}^{*}\left(b_{q}\right), a_{k}^{*}\left(a_{k}\right), \varepsilon_{k}, \varepsilon_{q}, g, C\left(\boldsymbol{k}_{1}, \boldsymbol{k}_{2}\right)$ and $D\left(\boldsymbol{k}_{1}, \boldsymbol{k}_{2}\right)$ in Eqs. (3a)-(3d) are creation (annihilation) operator of electron, creation (annihilation) operator of phonon, phonon frequency (in energy unit), electron band energy, electron-phonon coupling constant, mass change and force constant change parameters, respectively [40-49].

Let us take the following phonon Green function [50]:

$$
\begin{aligned}
& G_{k k^{\prime}}\left(t, t^{\prime}\right)=\left\langle\left\langle A_{k}(t) ; A_{k^{\prime}}^{*}\left(t^{\prime}\right)\right\rangle\right\rangle=-\mathrm{i} \theta\left(t-t^{\prime}\right) \\
& \quad \times\left[\left\langle A_{k}(t), A_{k^{\prime}}^{*}\left(t^{\prime}\right)\right\rangle\right],
\end{aligned}
$$

where $\theta\left(t-t^{\prime}\right)$ is the Heaviside unit step function.

Let us use equation of motion technique of quantum dynamics and the Dyson equation approach, Fourier transformed phonon Green function is obtained as $[13,34,47,48,50]$ :

$$
G_{k k^{\prime}}(\varepsilon)=\pi^{-1} \eta_{k k^{\prime}} \varepsilon_{k} /\left[\varepsilon^{2}-\bar{\varepsilon}_{k}^{2}+2 \mathrm{i} \varepsilon_{k} \Gamma(k q, \varepsilon)\right],
$$
where

$$
\begin{aligned}
& \eta_{k k^{\prime}}=\delta_{k k^{\prime}}+4 C\left(-\boldsymbol{k}, \boldsymbol{k}^{\prime}\right) \varepsilon_{k}^{-1}, \\
& \bar{\varepsilon}_{k}^{2}=\tilde{\varepsilon}_{k}^{2}+2 \varepsilon_{k} \Delta(k q, \varepsilon), \\
& \tilde{\varepsilon}_{k}^{2}=\varepsilon_{k}^{2}+\varepsilon_{k}(2 \pi)^{-1}\left(\varepsilon_{k}^{\mathrm{D}}+\varepsilon_{k}^{\mathrm{ep}}\right) .
\end{aligned}
$$

In Eq. $(6 \mathrm{c}), \varepsilon_{k}^{\mathrm{D}}$ and $\varepsilon_{k}^{\mathrm{ep}}$ are given as

$$
\begin{aligned}
\varepsilon_{k}^{\mathrm{D}} & =8 \pi\left[1+4 \varepsilon_{k}^{-1} \sum_{k_{1}} C\left(-\boldsymbol{k},-\boldsymbol{k}_{1}\right)\right]\left[\varepsilon_{k^{\prime}} \varepsilon_{k}^{-1} C\left(-\boldsymbol{k},-\boldsymbol{k}^{\prime}\right)\right. \\
& +D\left(-\boldsymbol{k},-\boldsymbol{k}^{\prime}\right)+4 \sum_{k^{\prime \prime}} C\left(-\boldsymbol{k},-\boldsymbol{k}^{\prime}\right) \\
& \left.\times D\left(-\boldsymbol{k}^{\prime \prime},-\boldsymbol{k}^{\prime}\right) \varepsilon_{k}^{-1}\right]+8 C\left(-\boldsymbol{k}, \boldsymbol{k}^{\prime}\right) \\
& \times\left(\varepsilon^{2}-\varepsilon_{k}^{2}\right) \varepsilon_{k}^{-1}, \\
\varepsilon_{k}^{\mathrm{ep}} & =4 \pi g\left[\left(\varepsilon_{k}^{-1} \varepsilon_{q}-g\right)\right. \\
& \left.\times\left(N_{q}+N_{Q}\right)+2 g \varepsilon_{k}^{-1}\left(N_{q k}+N_{Q k}\right)\right],
\end{aligned}
$$

where

$$
\begin{aligned}
& N_{q}=\left\langle b_{q}^{*} b_{q}\right\rangle, N_{Q}=\left\langle b_{Q}^{*} b_{Q}\right\rangle, N_{q k}=\left\langle b_{q}^{*} b_{q} B_{k}\right\rangle, \\
& N_{Q k}=\left\langle b_{Q}^{*} b_{Q} B_{k}\right\rangle .
\end{aligned}
$$

In above Eq. (5) and Eq. (6b), $\bar{\varepsilon}_{k}, \quad \Gamma(k q, \varepsilon), \quad \tilde{\varepsilon}_{k}$, and $\Delta(k q, \varepsilon)$ are perturbed mode frequency, phonon linewidth, renormalized mode frequency, and phonon shift, respectively. In Eq. (6c), D and ep are due to contributions from defect and electron phonon interaction parts, respectively. The response function is obtained as

$$
P(k q, \varepsilon)=P^{\mathrm{D}}(k q, \varepsilon)+P^{\mathrm{ep}}(k q, \varepsilon),
$$

where

$$
\begin{aligned}
& P^{\mathrm{D}}(k q, \varepsilon)=8 \sum_{k_{1}} R\left(-\boldsymbol{k}, \boldsymbol{k}_{1}\right) R^{*}\left(-\boldsymbol{k}, \boldsymbol{k}_{1}\right) \\
& \quad \times \varepsilon_{k_{1}}\left(\varepsilon^{2}-\tilde{\varepsilon}_{k_{1}}^{2}\right)^{-1}, \\
& P^{\mathrm{ep}}(k q, \varepsilon)=-\left[4 g^{2}\left(N_{q}+N_{Q} \delta_{Q Q^{\prime}}\right) /\left(\varepsilon-2 \varepsilon_{q}\right)\right] \\
& \quad \times\left(\varepsilon_{q}^{2}+g^{2} \tilde{n}_{k} \delta_{k k^{\prime}}\right) .
\end{aligned}
$$

Phonon linewidth and phonon shift are connected through response function $P(k q, \varepsilon)$ as

$$
P(k q, \varepsilon+\mathrm{i} \omega)=\Delta(k q, \varepsilon)-\mathrm{i} \Gamma(k q, \varepsilon), \omega \rightarrow 0^{+} .
$$

Phonon shift $\Delta(k q, \varepsilon)$ is given as 
$\Delta(k q, \varepsilon)=\Delta^{\mathrm{D}}(k q, \varepsilon)+\Delta^{\mathrm{ep}^{\prime}}(k q, \varepsilon)+\Delta^{\mathrm{ep}^{\prime \prime}}(k q, \varepsilon),(12)$ where

$$
\begin{aligned}
& \Delta^{\mathrm{D}}(k q, \varepsilon)=8 \mathrm{P} \sum_{k_{1}} R\left(-\boldsymbol{k}, \boldsymbol{k}_{1}\right) \\
& \quad \times R^{*}\left(-\boldsymbol{k}, \boldsymbol{k}_{1}\right) \varepsilon_{k_{1}}\left(\varepsilon^{2}-\tilde{\varepsilon}_{k_{1}}^{2}\right)^{-1}, \\
& \Delta^{\mathrm{ep}^{\prime}}(k q, \varepsilon)=\mathrm{P}\left[-4 g^{2} N_{q} /\left(\varepsilon-2 \varepsilon_{q}\right)\right] \\
& \quad \times\left(\varepsilon_{q}^{2}+g^{2} \tilde{n}_{k} \delta_{k k^{\prime}}\right), \\
& \Delta^{\mathrm{ep}^{\prime \prime}}(k q, \varepsilon)=\mathrm{P}\left[-4 g^{2} N_{Q} \delta_{Q Q^{\prime}} /\left(\varepsilon-2 \varepsilon_{q}\right)\right] \\
& \quad \times\left(\varepsilon_{q}^{2}+g^{2} \tilde{n}_{k} \delta_{k k^{\prime}}\right) .
\end{aligned}
$$

In Eqs. (13a)-(13c), "P" represents principal value. Phonon linewidth $\Gamma(k q, \varepsilon)$ is given as

$$
\Gamma(k q, \varepsilon)=\Gamma^{\mathrm{D}}(k q, \varepsilon)+\Gamma^{\mathrm{ep}^{\prime}}(k q, \varepsilon)+\Gamma^{\mathrm{ep}^{\prime \prime}}(k q, \varepsilon),(14)
$$
where

$$
\begin{aligned}
& \Gamma^{\mathrm{D}}(k q, \varepsilon)=8 \pi \omega(\varepsilon) \sum_{k_{1}} R\left(-\boldsymbol{k}, \boldsymbol{k}_{1}\right) \\
& \quad \times R^{*}\left(-\boldsymbol{k}, \boldsymbol{k}_{1}\right) \varepsilon_{k_{1}} \delta\left(\varepsilon^{2}-\tilde{\varepsilon}_{k_{1}}^{2}\right), \\
& \Gamma^{\mathrm{ep}^{\prime}}(k q, \varepsilon)=4 g^{2} N_{q}\left(\varepsilon_{q}^{2}+g^{2} \tilde{n}_{k} \delta_{k k^{\prime}}\right) \delta\left(\varepsilon-2 \varepsilon_{q}\right), \\
& \Gamma^{\mathrm{ep}^{\prime \prime}}(k q, \varepsilon)=4 g^{2} N_{Q} \delta_{Q Q^{\prime}} \\
& \quad \times\left(\varepsilon_{q}^{2}+g^{2} \tilde{n}_{k} \delta_{k k^{\prime}}\right) \delta\left(\varepsilon-2 \varepsilon_{q}\right) .
\end{aligned}
$$

$N_{q}, N_{Q}, \tilde{n}_{k}$ of Eqs. (10b, 13b, 13c, 15b, 15c) are evaluated by applying equation of motion technique of quantum dynamics to find the newly Fourier transformed phonon Green function $G_{k k^{\prime}}(\varepsilon)$ as [13, 34, 47, 48, 50]:

$$
\begin{gathered}
G_{k k^{\prime}}(\varepsilon)=\varepsilon_{k} \pi^{-1}\left[1+4 C\left(\boldsymbol{k}^{\prime},-\boldsymbol{k}\right) \varepsilon_{k}^{-1}\right]\left(\varepsilon^{2}-\tilde{\varepsilon}_{k}^{\prime 2}\right)^{-1} \\
-4 g\left(\varepsilon^{2}-\tilde{\varepsilon}_{k}^{2}\right)^{-1} \sum_{q}\left[\varepsilon_{q} G_{q^{\prime} q k^{\prime}}(\varepsilon)+g G_{k q^{\prime} q k^{\prime}}(\varepsilon)\right],
\end{gathered}
$$

where

$$
\begin{aligned}
\tilde{\varepsilon}_{k}^{\prime 2} & =\varepsilon_{k}^{2}+4 \varepsilon_{k} \sum_{k_{1}}\left[C\left(\boldsymbol{k}_{1},-\boldsymbol{k}\right)+D\left(\boldsymbol{k}_{1},-\boldsymbol{k}_{1}\right)\right. \\
& \left.+4 C\left(\boldsymbol{k}_{1},-\boldsymbol{k}\right) D\left(\boldsymbol{k}_{1},-\boldsymbol{k}_{1}\right)\right] .
\end{aligned}
$$

The Green functions $G_{q^{\prime} q k^{\prime}}(\varepsilon)=\left\langle\left\langle b_{q^{\prime}}^{*} b_{q} ; A_{k^{\prime}}\right\rangle\right\rangle$ and $G_{k q^{\prime} q k^{\prime}}(\varepsilon)=\left\langle\left\langle B_{k} b_{q^{\prime}}^{*} b_{q} ; A_{k^{\prime}}\right\rangle\right\rangle$ of Eq. (16) can be obtained again by using equation of motion technique of quantum dynamics $[13,34,47,48,50]$ and they are substituted in Eq. (16) to get

$$
\begin{aligned}
& G_{k k^{\prime}}(\varepsilon)=2^{-1} \pi^{-1} \varepsilon_{k} \tilde{\varepsilon}_{k}^{\prime-1}\left[1+4 C\left(\boldsymbol{k}^{\prime},-\boldsymbol{k}\right) \varepsilon_{k}^{-1}\right] \\
& \quad \times\left[\left(\varepsilon-\tilde{\varepsilon}_{k}^{\prime}\right)^{-1}-\left(\varepsilon+\tilde{\varepsilon}_{k}^{\prime}\right)^{-1}\right]-2 \pi^{-1} g^{2} \sum_{q} N_{q} \\
& \quad \times\left\{\left[\left(\tilde{\varepsilon}_{k}^{\prime}-2 \varepsilon_{q}\right)^{2}-\varepsilon_{k}^{2}\right]^{-1}\left(\varepsilon-\tilde{\varepsilon}_{k}^{\prime}\right)^{-1}\right. \\
& \quad+\left[\left(\tilde{\varepsilon}_{k}^{\prime}+2 \varepsilon_{q}\right)^{2}-\varepsilon_{k}^{2}\right]^{-1}\left(\varepsilon+\tilde{\varepsilon}_{k}^{\prime}\right)^{-1}+\left(\varepsilon_{k}-2 \varepsilon_{q}\right) \varepsilon_{k}^{-1} \\
& \quad \times\left[\left(\varepsilon_{k}-2 \varepsilon_{q}\right)^{2}-\tilde{\varepsilon}_{k}^{2}\right]^{-1}\left[\left(\varepsilon-2 \varepsilon_{q}\right)+\varepsilon_{k}\right]^{-1}+\left(\varepsilon_{k}+2 \varepsilon_{q}\right) \\
& \left.\quad \times \varepsilon_{k}^{-1}\left[\left(\varepsilon_{k}+2 \varepsilon_{q}\right)^{2}-\varepsilon_{k}^{\prime 2}\right]^{-1}\left[\left(\varepsilon-2 \varepsilon_{q}\right)-\varepsilon_{k}\right]^{-1}\right\} . \quad(18)
\end{aligned}
$$

The above evaluated phonon Green function Eq. (18), gives $N_{Q}, N_{q}$ and $\tilde{n}_{k}$ of Eqs. $(10 \mathrm{~b}, 13 \mathrm{~b}, 13 \mathrm{c}, 15 \mathrm{~b}, 15 \mathrm{c})$ as [51]:

$$
\begin{aligned}
& \tilde{n}_{k}=64 \pi^{-1} g^{2} k_{\mathrm{B}}^{2} T^{2} \varepsilon_{k} \tilde{\varepsilon}_{k}^{\prime-2}\left[1+4 C\left(\boldsymbol{k}^{\prime},-\boldsymbol{k}\right) \varepsilon_{k}^{-1}\right] \\
& \quad \times \sum_{q} \varepsilon_{q}^{2} A_{k Q}\left(\tilde{\varepsilon}_{k}^{\prime 2}-\tilde{\varepsilon}_{Q}^{\prime 2}\right)^{2}\left(\varepsilon_{k}^{4}-8 \varepsilon_{k}^{2} \varepsilon_{q}^{2}-2 \varepsilon_{k}^{2} \tilde{\varepsilon}_{k}^{2}\right. \\
& \left.\quad-8 \tilde{\varepsilon}_{k}^{2} \varepsilon_{q}^{2}+16 \varepsilon_{q}^{4}+\tilde{\varepsilon}_{k}^{\prime 4}\right) C_{k q k}(-) C_{k q k}(+) \\
& \quad \times D_{k q k}(-) D_{k q k}(+), \\
& N_{q}=2 \pi^{-1} k_{\mathrm{B}} T \varepsilon_{k} \tilde{\varepsilon}_{k}^{-2}\left[1+4 C\left(\boldsymbol{k}^{\prime},-\boldsymbol{k}\right) \varepsilon_{k}^{-1}\right] \\
& \quad \times A_{k q} B_{k q} C_{k q k}(-) C_{k q k}(+) D_{k q k}(-) D_{k q k}(+) \\
& \quad \times E_{k q q}(-) E_{k q q}(+), \\
& N_{Q}=2 \pi^{-1} k_{\mathrm{B}} T \varepsilon_{k} \tilde{\varepsilon}_{k}^{\prime-2}\left[1+4 C\left(\boldsymbol{k}^{\prime},-\boldsymbol{k}\right) \varepsilon_{k}^{-1}\right] \\
& \quad \times A_{k Q} B_{k Q} C_{k q k}(-) C_{k q k}(+) \\
& \quad \times D_{k q k}(-) D_{k q k}(+) E_{k q Q}(-) E_{k q Q}(+),
\end{aligned}
$$
where

$$
\begin{gathered}
A_{k m}=\left(\tilde{\varepsilon}_{k}^{\prime 2}+\tilde{\varepsilon}_{m}^{\prime 2}\right), \quad B_{k m}=\left(\tilde{\varepsilon}_{k}^{\prime 2}-\tilde{\varepsilon}_{m}^{\prime 2}\right)^{-4}, \\
m=q, Q \\
C_{k q k}(\mp)=\left[\left(\tilde{\varepsilon}_{k}^{\prime} \mp 2 \varepsilon_{q}\right)^{2}-\varepsilon_{k}^{2}\right]^{-1}, \\
D_{k q k}(\mp)=\left[\left(\varepsilon_{k} \mp 2 \varepsilon_{q}\right)^{2}-\tilde{\varepsilon}_{k}^{\prime 2}\right]^{-1},
\end{gathered}
$$

$E_{k q m}(\mp)=\left[\left(\varepsilon_{k} \mp 2 \varepsilon_{q}\right) \pm \tilde{\varepsilon}_{m}^{\prime}\right]^{-2}, \quad m=q, Q$.

Equations (15b) and (15c), with the help of Eqs. (19a)(19c), are modified as

$$
\begin{aligned}
& \Gamma^{\mathrm{ep}^{\prime}}(k q, \varepsilon)=\left\{g^{2} T\left[1+4 C\left(\boldsymbol{k}^{\prime},-\boldsymbol{k}\right) \varepsilon_{k}^{-1}\right]\right. \\
&+g^{6} T^{3}\left[1+16\left(C\left(\boldsymbol{k}^{\prime},-\boldsymbol{k}\right)\right)^{2} \varepsilon_{k}^{-2}+8 C\left(\boldsymbol{k}^{\prime},-\boldsymbol{k}\right) \varepsilon_{k}^{-1}\right] \\
&\times I(k q k Q)\} A_{k q} B_{k q} F(k q k) E_{k q q}(-) E_{k q q}(+) \\
& \times \delta\left(\varepsilon-2 \varepsilon_{q}\right), \\
& \Gamma^{\mathrm{ep}^{\prime \prime}}(k q, \varepsilon)=\left\{g^{2} T\left[1+4 C\left(\boldsymbol{k}^{\prime},-\boldsymbol{k}\right) \varepsilon_{k}^{-1}\right]\right. \\
& \quad+g^{6} T^{3}\left[1+16\left(C\left(\boldsymbol{k}^{\prime},-\boldsymbol{k}\right)\right)^{2} \varepsilon_{k}^{-2}+8 C\left(\boldsymbol{k}^{\prime},-\boldsymbol{k}\right) \varepsilon_{k}^{-1}\right] \\
&\quad \times I(k q k Q)\} A_{k Q} B_{k Q} F(k q k) E_{k q Q}(-) E_{k q Q}(+) \\
& \quad \times \delta\left(\varepsilon-2 \varepsilon_{q}\right),
\end{aligned}
$$

where

$$
\begin{aligned}
& F(k q k)=8 \pi^{-1} k_{\mathrm{B}} \varepsilon_{k} \tilde{\varepsilon}_{k}^{\prime-2} \varepsilon_{q}^{2} C_{k q k}(-) C_{k q k}(+) \\
& \quad \times D_{k q k}(-) D_{k q k}(+), \\
& I(k q k Q)=64 \pi^{-1} k_{\mathrm{B}}^{2} \tilde{\varepsilon}_{k}^{\prime-2} \sum_{q} \varepsilon_{k} A_{k Q}\left(\tilde{\varepsilon}_{k}^{\prime 2}-\tilde{\varepsilon}_{Q}^{\prime 2}\right)^{2} \\
& \quad \times\left(\varepsilon_{k}^{4}-8 \varepsilon_{k}^{2} \varepsilon_{q}^{2}-2 \varepsilon_{k}^{2} \tilde{\varepsilon}_{k}^{\prime 2}-8 \tilde{\varepsilon}_{k}^{\prime 2} \varepsilon_{q}^{2}+16 \varepsilon_{q}^{4}+\tilde{\varepsilon}_{k}^{\prime 4}\right) \\
& \quad \times C_{k q k}(-) C_{k q k}(+) D_{k q k}(-) D_{k q k}(+) .
\end{aligned}
$$




\section{Evaluation of renormalized mode frequency and the Debye-Waller factor}

The substitution of Eq. (19b) and Eq. (19c) in Eq. (6c) yields the renormalized mode frequency in low impurity concentration as

$$
\tilde{\varepsilon}_{k}^{2}=\left(\tilde{\varepsilon}_{k}^{2}\right)^{\prime}+\left(\tilde{\varepsilon}_{k}^{2}\right)^{\prime \prime},
$$

where

$$
\begin{aligned}
& \left(\tilde{\varepsilon}_{k}^{2}\right)^{\prime}=\varepsilon_{k}^{2}+\varepsilon_{k}(2 \pi)^{-1}\left[\varepsilon_{k}^{\mathrm{D}}+8 g k_{\mathrm{B}} T \varepsilon_{k}^{-1}\left(\varepsilon_{k}^{-1} \varepsilon_{q}-g\right)\right. \\
& \quad \times C_{k q k}(-) C_{k q k}(+) D_{k q k}(-) D_{k q k}(+) \\
& \quad \times\left(A_{k q} B_{k q} E_{k q q}(-) E_{k q q}(+)\right. \\
& \left.\left.\quad+A_{k Q} B_{k Q} E_{k q Q}(-) E_{k q Q}(+)\right)\right], \\
& \left(\tilde{\varepsilon}_{k}^{2}\right)^{\prime \prime}=\varepsilon_{k}^{2}+\varepsilon_{k}(2 \pi)^{-1}\left[\varepsilon_{k}^{\mathrm{D}}+32 k_{\mathrm{B}} g T C\left(\boldsymbol{k}^{\prime},-\boldsymbol{k}\right) \varepsilon_{k}^{-2}\right. \\
& \quad \times\left(\varepsilon_{k}^{-1} \varepsilon_{q}-g\right) C_{k q k}(-) C_{k q k}(+) D_{k q k}(-) D_{k q k}(+) \\
& \quad \times\left(A_{k q} B_{k q} E_{k q q}(-) E_{k q q}(+)\right. \\
& \left.\left.\quad+A_{k Q} B_{k Q} E_{k q Q}(-) E_{k q Q}(+)\right)\right] .
\end{aligned}
$$

Equation (24a) and Eq. (24b) are the diagonal and nondiagonal parts of renormalized mode frequency, respectively. The Debye-Waller factor is obtained after substituting imaginary part of phonon Green function Eq. (5) in Eq. (1) through Eqs. (14, 15a, 21a, 21b) as [13]:

$$
\begin{aligned}
& {[2 W]=[2 W]_{d}^{\mathrm{D}}+[2 W]_{n d}^{\mathrm{D}}+[2 W]_{d}^{\mathrm{ep}^{\prime}}+[2 W]_{n d}^{\mathrm{ep}}} \\
& \quad+[2 W]_{d}^{\mathrm{ep} "}+[2 W]_{n d}^{\mathrm{ep} "}
\end{aligned}
$$

where,

$$
\begin{aligned}
& {[2 W]_{d}^{\mathrm{D}}=16 \pi \hbar^{-1} k_{\mathrm{B}} T(M N)^{-1} \sum_{k, k_{1}} K^{2} \omega\left(\tilde{\varepsilon}_{k_{1}}^{2}\right) \varepsilon_{k} \tilde{\varepsilon}_{k_{1}}^{-2}} \\
& \quad \times R\left(\boldsymbol{k}_{1},-\boldsymbol{k}\right) R^{*}\left(\boldsymbol{k}_{1},-\boldsymbol{k}\right)\left(\tilde{\varepsilon}_{k_{1}}^{2}-\bar{\varepsilon}_{k}^{2}\right)^{-2}, \quad(26 \mathrm{a}) \\
& {[2 W]_{n d}^{\mathrm{D}}=64 \pi \hbar^{-1} k_{\mathrm{B}} T(M N)^{-1} \sum_{k, k^{\prime}, k_{1}} C\left(-\boldsymbol{k}, \boldsymbol{k}^{\prime}\right)} \\
& \quad \times[\boldsymbol{K} \cdot \boldsymbol{e}(k)]\left[\boldsymbol{K} \cdot \boldsymbol{e}\left(k^{\prime}\right)\right] \omega\left(\tilde{\varepsilon}_{k_{1}}^{2}\right) \tilde{\varepsilon}_{k_{1}}^{-2} R\left(\boldsymbol{k}_{1},-\boldsymbol{k}\right) \\
& \quad \times R^{*}\left(\boldsymbol{k}_{1},-\boldsymbol{k}\right)\left(\tilde{\varepsilon}_{k_{1}}^{2}-\bar{\varepsilon}_{k}^{2}\right)^{-2}, \\
& {[2 W]_{d}^{\mathrm{ep}}{ }^{\prime}=\hbar^{-1} k_{\mathrm{B}} g^{2} T^{2}(M N)^{-1} \sum_{k} K^{2} \varepsilon_{k} \varepsilon_{q}^{-1}} \\
& \quad \times\left(4 \varepsilon_{q}^{2}-\bar{\varepsilon}_{k}^{2}\right)^{-2} F(k q k) A_{k q} B_{k q} E_{k q q}(-) E_{k q q}(+) \\
& \quad \times\left[1+g^{4} T^{2} I(k q k Q)\right], \\
& {[2 W]_{n d}^{\mathrm{ep}}=4 \hbar^{-1} k_{\mathrm{B}} g^{2} T^{2}(M N)^{-1} \sum_{k, k^{\prime}} C\left(-\boldsymbol{k}, \boldsymbol{k}^{\prime}\right)} \\
& \quad \times[\boldsymbol{K} \cdot \boldsymbol{e}(k)]\left[\boldsymbol{K} \cdot \boldsymbol{e}\left(k^{\prime}\right)\right] \varepsilon_{q}^{-1}\left(4 \varepsilon_{q}^{2}-\bar{\varepsilon}_{k}^{2}\right)^{-2} F(k q k) \\
& \quad \times A_{k q} B_{k q} E_{k q q}(-) E_{k q q}(+)\left[1+4 C\left(\boldsymbol{k}^{\prime},-\boldsymbol{k}\right)\right. \\
& \left.\quad \times g^{4} T^{2} I(k q k Q)\right], \\
& {[2 W]_{d}^{\mathrm{ep}}{ }^{\prime \prime}=\hbar^{-1} k_{\mathrm{B}} g^{2} T^{2}(M N)^{-1} \sum_{k} K^{2} \varepsilon_{k} \varepsilon_{q}^{-1}} \\
& \quad \times\left(4 \varepsilon_{q}^{2}-\bar{\varepsilon}_{k}^{2}\right)^{-2} F(k q k) A_{k Q} B_{k Q} E_{k q Q}(-) E_{k q Q}(+)
\end{aligned}
$$

$$
\begin{aligned}
& \quad \times\left[1+g^{4} T^{2} I(k q k Q)\right], \\
& {[2 W]_{n d}^{\mathrm{ep}^{\prime \prime}}=4 \hbar^{-1} k_{\mathrm{B}} g^{2} T^{2}(M N)^{-1} \sum_{k, k^{\prime}} C\left(-k, k^{\prime}\right)} \\
& \times[\boldsymbol{K} \cdot \boldsymbol{e}(k)]\left[\boldsymbol{K} \cdot \boldsymbol{e}\left(k^{\prime}\right)\right] \varepsilon_{q}^{-1}\left(4 \varepsilon_{q}^{2}-\bar{\varepsilon}_{k}^{2}\right)^{-2} F(k q k) \\
& \quad \times A_{k Q} B_{k Q} E_{k q Q}(-) E_{k q Q}(+)\left[1+4 C\left(\boldsymbol{k}^{\prime},-\boldsymbol{k}\right)\right. \\
& \left.\quad \times g^{4} T^{2} I(k q k Q)\right] .
\end{aligned}
$$

In Eq. (25) and Eqs. (26a)-(26f), $d$ and $n d$ are diagonal and non-diagonal terms of defect $(D)$ and electronphonon interaction parts $\left(\mathrm{ep}^{\prime}, \mathrm{ep}^{\prime \prime}\right)$ of DWF, respectively.

\section{Results and discussions}

Phonon linewidth obtained from equation of motion technique provide defect and electron-phonon interaction contributions in semiconductor crystals. They are responsible for diagonal and non-diagonal contribution to DWF. The perturbed mode frequency is contributed by renormalized mode frequency and phonon shift. Renormalized mode frequency and phonon shift are contributed by defect dependent term and electronphonon interaction term. The renormalized mode frequency is obtained in terms of diagonal and non-diagonal parts. The defect part of it is dependent on mass and force constant change parameters and their cross-term while electron-phonon interaction part is linear and nonlinear function of electron-phonon coupling constant and linear variation on temperature. The formation of localized mode and an interaction of electron with phonon are the source to generate renormalized localized mode frequency and two exciton bound state, respectively. When two exciton bound state and renormalized localized mode frequency are identical with perturbed mode frequency, the intensity of the Debye-Waller factor increases asymptotically. An electron-phonon interaction shows the dominant contribution through linear and nonlinear dependence on mass change parameter in comparison to linear variation of defect term on mass change parameter in the Debye-Waller factor. It is evident in semiconductor that $T^{2}, T^{4}$ dependence due to electronphonon interaction is dominant over linear dependence on temperature in localized field. In low impurity concentration limit, the diagonal part gives its more contribution in comparison to non-diagonal part for this dynamical property.

In low temperature limit, $T^{4}$ dependence is dominant over $T^{2}$ dependent term due to strong coupling constant $g^{6}$ in comparison to $g^{2}$ term. The temperature dependence of DWF can also be seen in Ref. [52]. In the absence of electron-phonon interaction and also in the absence of low temperature limit, this work tends to defect dependent term of the Debye-Waller factor of Ref. [13]. It is also found from this theory that weak electron-phonon coupling demands low power dependence on mass change parameter in comparison to high power dependence on mass change parameter in strong electron-phonon coupling. The terms $B_{k q}, B_{k Q}$, 
$C_{k q k}(\mp), D_{k q k}(\mp), E_{k q q}(\mp), E_{k q Q}(\mp)$ provide the significant contribution in the limit $\tilde{\varepsilon}_{k}^{\prime}=\tilde{\varepsilon}_{q}^{\prime}, \tilde{\varepsilon}_{Q}^{\prime}, \tilde{\varepsilon}_{k}^{\prime} \mp 2 \varepsilon_{q}=$ $\varepsilon_{k}, \varepsilon_{k} \mp 2 \varepsilon_{q}=\tilde{\varepsilon}_{k}^{\prime}, \varepsilon_{k} \mp 2 \varepsilon_{q}=\mp \tilde{\varepsilon}_{q}^{\prime}$, $\mp \tilde{\varepsilon}_{Q}^{\prime}$ respectively to increase the DWF. In low temperature limit, an interaction of electron with harmonic and localized fields are the major factors to explain the temperature dependence of DWF as obtained by previous authors adopting a different technique [52]. This paper is limited to develop the theory to know the cause of temperature dependence of DWF by applying equation of motion technique of quantum dynamics, and the Dyson equation approach, so numerical results have not been evaluated.

\section{Conclusion}

The renormalized mode frequency, and the DebyeWaller factor are expressed in terms of diagonal and nondiagonal parts. Renormalized mode frequency is linearly and non-linearly dependent on electron phonon coupling constant, and linearly dependent on temperature. But DWF is non-linearly dependent on both electron-phonon coupling constants and temperature in electron-phonon interaction field in semiconductor crystals. The nondiagonal parts are dependent on mass change parameter, and vanish in absence of impurity. The creation of renormalized localized mode frequency, perturbed mode frequency, and two exciton bound state are the source to influence the intensity of the Debye-Waller factor.

\section{References}

[1] R. Ishikawa, R. Mishra, A.R. Lupini, S.D. Findlay, T. Taniguchi, S.T. Pantelides, S.J. Pennycook, Phys. Rev. Lett. 113, 155501 (2014).

[2] B. Saikia, A. Kumar, Pramana J. Phys. 71, 143 (2008).

[3] J.E. Anthony, A. Facchetti, M. Heeney, S.R. Marder, X. Zhan, Adv. Mater. 22, 3876 (2010).

[4] B. Lal, P. Aghamkar, S. Kumar, M.K. Kashyap, Eur. Phys. J. D 61, 717 (2011).

[5] D.W. Snoke, Ann. Phys. 523, 87 (2011).

[6] S. Kumar, M. Khorasaninejad, M.M. Adachi, K.S. Karim, S.S. Saini, A.K. Sood, Pramana J. Phys. 79, 471 (2012).

[7] Y. Ezzahri, K. Joulain, J. Appl. Phys. 112, 083515 (2012).

[8] H. Shimizu, S. Umetsu, T. Kaihara, Jpn. J. Appl. Phys. 52, 028006 (2013).

[9] T.T. Zhang, P. Barate, C.T. Nguyen, A. Balocchi, T. Amand, P. Renucci, H. Carrere, B. Urbaszek, X. Marie, Phys. Rev. B 87, 041201(R) (2013).

[10] Xin Chen, D. Parker, D.J. Singh, Phys. Rev. B 87, 045317 (2013).

[11] N. Rani, M. Yadav, Y.K. Mathur, Int. J. Appl. Eng. Res. 9, 59 (2014).

[12] A.A. Maradudin, A.E. Fein, Phys. Rev. 128, 2589 (1962).

[13] B.P. Bahuguna, C.P. Painuli, B.D. Indu, Int. J. Theor. Phys. 30, 807 (1991).
[14] H.K. Hieu, V.V. Hung, N.V. Hung, Proc. Natl. Conf. Theor. Phys. 35, 109 (2010).

[15] J. Callaway, Quantum Theory of the Solid State (Part A), Academic Press, New York 1974, Ch. 1, p. 46.

[16] M.S. Kushwaha, Phys. Rev. B 24, 2115 (1981).

[17] S.G. Sahaya Sheba, K. Sadaiyandi, Int. J. Pure Appl. Phys. 7, 139 (2011).

[18] H.G. Bohn, W. Zinn, F. Tasset, J. Phys. Coll. 43 C7, 141 (1982).

[19] Sang-Wook Han, Jpn. J. Appl. Phys. 42 10, 6303 (2003).

[20] D.Y. Song, M. Basavaraj, S.A. Nikishin, M. Holtz, V. Soukhoveev, A. Usikov, V. Dmitriev, J. Appl. Phys. 100, 113504 (2006).

[21] N.V. Hung, N.C. Toan, N.B. Duc, D.Q. Vuong, Open Phys. 13, 242 (2015).

[22] Z.M. Hund, K.J. Nihill, D. Campi, K.T. Wong, N.S. Lewis, M. Bernasconi, G. Benedek, S.J. Sibener, J. Chem. Phys. 143, 124705 (2015).

[23] J.P. Rino, P.S. Pizani, S.C. Costa, Braz. J. Phys. 34, 347 (2004).

[24] M. Schowalter, A. Rosenauer, J.T. Titantah, D. Lamoen, Microsc. Microanal. 13 S03, 128 (2007).

[25] N.V. Hung, C.S. Thang, N.C. Toan, H.K. Hieu, Vacuum 101, 63 (2014).

[26] N.V. Hung, J.J. Rehr, Phys. Rev. B 56, 43 (1997).

[27] N.V. Hung, N.B. Duc, R.R. Frahm, J. Phys. Soc. Jpn. 72, 1254 (2003).

[28] N.V. Hung, N.B. Trung, B. Kirchner, Physica B 405 , 2519 (2010).

[29] N.V. Hung, T.S. Tien, N.B. Duc, D.Q. Vuong, Mod. Phys. Lett. B 28, 1450174 (2014).

[30] N.V. Hung, N.B. Trung, N.B. Duc, D.D. Son, T.S. Tien, J. Phys. Sci. Appl. 4, 43 (2014).

[31] A.A. Maradudin, in: Solid State Physics, Eds. F. Seitz, D. Turnbull, Academic Press, New York 1966, Vol. 18, p. 273.

[32] A.A. Maradudin, Astrophysics and The Many-Body Problem, Benjamin, New York 1963, p. 109.

[33] A.A. Maradudin, P. Mazur, E.W. Montroll, G.H. Weiss, Rev. Mod. Phys. 30, 175 (1958).

[34] B.D. Indu, Int. J. Mod. Phys. B 4, 1379 (1990).

[35] O. Madelung, in: Introduction to Solid-State Theory, Springer Series in Solid-State Sciences, Eds. M. Cardona, P. Fulde, H.-J. Queisser, Springer, Berlin 1978, Vol. 2, Ch. 4, p. 183.

[36] S.C. Gairola, Ind. J. Phys. 86, 967 (2012).

[37] C. Kittel, Quantum Theory of Solids, Wiley, New York 1963, Ch. 7.

[38] R.C. Shukla, E.R. Muller, Phys. Rev. B 21, 544 (1980).

[39] R.C. Shukla, Phys. Rev. B 22, 5810 (1980).

[40] A.A. Maradudin, E.W. Montroll, G.H. Weiss, I.P. Ipatova, Solid State Physics, Theory of Lattice Dynamics in the Harmonic Approximation, Suppl. 3, 2nd ed., Academic Press, New York 1971, Ch. 2, p. 56.

[41] H. Frohlich, in: New Perspectives in Modern Physics, Ed. R.E. Marshak, Wiley, New York 1966, p. 539. 
[42] H.Y. Fan, Elements of Solid State Physics, Wiley, New York 1987, p. 183 and 184.

[43] D. Feinberg, S. Ciuchi, F. de Pasquale, Int. J. Mod. Phys. B 4, 1317 (1990).

[44] G.C. Mahanty, S.N. Behera, Can. J. Phys. 61, 1160 (1983).

[45] S.N. Behera, S.G. Mishra, Phys. Rev. B 31, 2773 (1985).

[46] J.M. Ziman, Elements of Advanced Quantum Theory, Cambridge University Press, Cambridge 1969, Ch. 2, p. 38 and 43.

[47] P.K. Sharma, R. Bahadur, Phys. Rev. B 12, 1522 (1975).
[48] D.N. Sahu, P.K. Sharma, Phys. Rev. B 28, 3200 (1983).

[49] S. Srivastava, Y.P. Raiwani, S.C. Gairola, B.D. Indu, Physica B 223-224, 538 (1996).

[50] D.N. Zubarev, Usp. Fiz. Nauk 71, 71 (1960) [ Eng. Transl. Sov. Phys. Usp. 3, 320 (1960)].

[51] K.N. Pathak, Phys. Rev. 139, A1569 (1965).

[52] M. Schowalter, A. Rosenauer, J.T. Titantah, D. Lamoen, Acta Cryst. A 65, 227 (2009). 